\title{
Planetary nebulae in the GLIMPSE survey
}

\author{
Sun Kwok ${ }^{1}$, Nico Koning ${ }^{2}$, Hsiu-Hui Huang ${ }^{3}$ and Edward Churchwell ${ }^{4}$ \\ ${ }^{1}$ Department of Physics, University of Hong Kong, Hong Kong, China \\ email: sunkwok@hku.hk \\ ${ }^{2}$ Department of Physics \& Astronomy, University of Calgary, Calgary, Canada T2N 1N4 \\ ${ }^{3}$ Institute of Astronomy \& Astrophysics, Academia Sinica, Taipei, Taiwan \\ ${ }^{4}$ Department of Astronomy, University of Wisconsin, Madison, WI 53706, U.S.A.
}

\begin{abstract}
We report the observations of planetary nebulae in the Spitzer Space Telescope Galactic Legacy Infrared Mid-Plane Survey Extraordinaire (GLIMPSE) survey. The distribution of warm dust is clearly shown in these images.
\end{abstract}

Keywords. planetary nebulae: general, infrared: general, surveys

\section{Introduction}

Planetary nebulae (PNs) have high dust content and radiate strongly in the infrared. For young PNs, the dust component accounts for $\sim 1 / 3$ of the total energy output of the nebulae (Zhang \& Kwok 1991). The typical color temperatures of PNs are between 100 and $200 \mathrm{~K}$, and at $\lambda>5 \mu \mathrm{m}$, dust begins to dominate over bound-free emission from the ionized component. Although PNs are traditionally discovered through examination of photographic plates or $\mathrm{H} \alpha$ surveys, $\mathrm{PNs}$ can also be identified in infrared surveys by searching for red objects with a rising spectrum between $4-10 \mu \mathrm{m}$. In this paper, we present images of PNs observed in the Galactic Legacy Infrared Mid-Plane Survey Extraordinaire (GLIMPSE) survey, one of the Legacy Science Projects of the Spitzer Space Telescope (SST).

\section{Data processing}

The GLIMPSE survey was carried out over the period from March 9 to November 1 in 2004 with the Infrared Array Camera (IRAC) on the SST. IRAC has four wavelength bands centered at 3.6, 4.5, 5.8 and $8.0 \mu \mathrm{m}$, each of which has a field of view of $\sim 5.2^{\prime} \times 5.2^{\prime}$. The pixel size in all four bands is $\sim 1.2^{\prime \prime} \times 1.2^{\prime \prime}$. The FWHM of the point-spread functions are 1.63", 1.70", 1.85" and 1.94" at 3.6, 4.5, 5.8 and $8.0 \mu \mathrm{m}$ bands, respectively. The GLIMPSE survey covers the inner two-thirds of the Galactic plane $\left(\ell= \pm 10^{\circ}-65^{\circ}, b=\right.$ $\pm 1^{\circ}$; total area $\left.\sim 220 \mathrm{deg}^{2}\right)$ in all four IRAC bands. Details of the survey are described in Churchwell et al. (2004).

The GLIMPSE data processing effort starts from the basic calibrated data supplied by the Spitzer Space Center to produce independent mosaics using the Montage package (montage.ipac.caltech.edu). The mosaic images were corrected for instrumental artifacts such as zodiacal background, excessive cosmic ray hits and other instrumental and downlink problems. Counterparts of PNs from the Edinburgh/AAO/Strasbourg Catalogue of Galactic Planetary Nebulae were then searched for and identified in the GLIMPSE mosaics. 

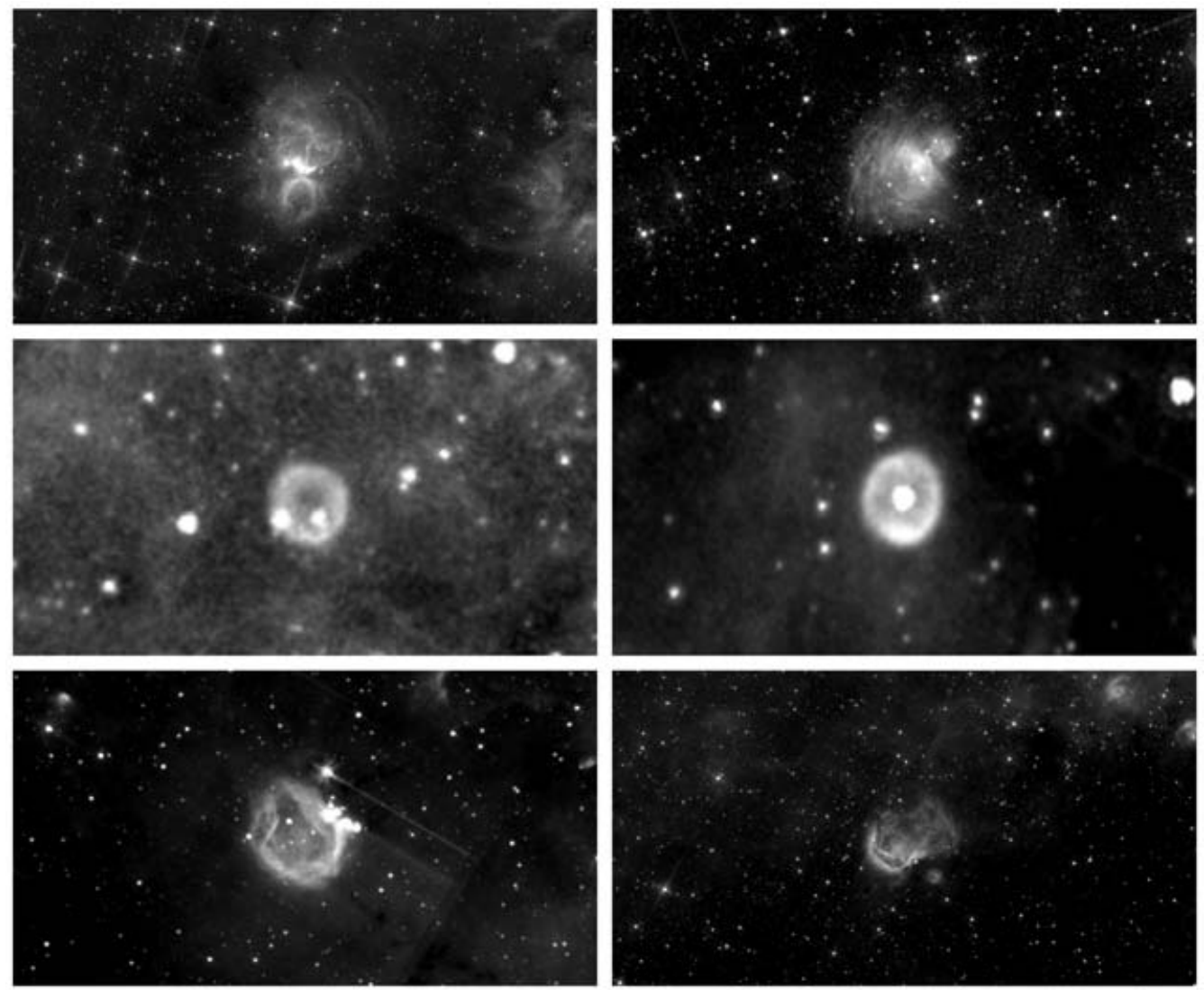

Figure 1. IRAC images of $6 \mathrm{PNs}$ observed in the GLIMPSE survey (upper left: PNG $328.5-00.5(5.8 \mu \mathrm{m})$, upper right: PNG 298.4+00.6 $(5.8 \mu \mathrm{m})$, middle left: PNG 040.3-00.4 $(8 \mu \mathrm{m})$, middle right: PNG 333.9+00.6 $(8 \mu \mathrm{m})$, lower left: PNG 321.3-00.3 $(8 \mu \mathrm{m})$, lower right: PNG $309.5-00.7(5.8 \mu \mathrm{m})$.

\section{Results}

A total of 31 counterparts to PNs were found in the GLIMPSE survey. Most are southern PNs catalogued by the AAO/UKAST H $\alpha$ survey. Many of the PNs have a rising spectrum in the $3-8 \mu \mathrm{m}$ range and are brightest in the $8 \mu \mathrm{m}$ band. The images of six PNs are shown in Figure 1. Since many HiI regions are also dusty, we cannot be certain that these GLIMPSE sources are PNs. Their nature has to be followed up by ground-based optical imaging and spectroscopy.

We can see that the infrared morphologies range from typical shell-like structures (e.g., PNG 040.3-00.4, PNG 321.3-00.3) to bipolar structures (e.g., PNG 298.4+00.6). In the case of PNG 328.5-00.4, the two bipolar lobes are bright in the IRAC images, showing that there is dust emission in the lobes. However, the two lobes are separated by a dark lane, which must contain dust too cold to radiate at $8 \mu \mathrm{m}$. The distribution of such cold dust in the equatorial regions needs to be imaged by a far-infrared imager, e.g., with the FORCAST instrument of SOFIA or submm interferometers such as ALMA.

\section{References}

Churchwell, E. B., et al. 2004, ApJS 154, 322

Zhang, C.Y. \& Kwok, S. 1991, A\&SA 250, 179 